\title{
3 Sprachen als Ressourcen, Sprachen als Produkte auf einem Markt: Hintergründe, Widersprüche und Grenzen
}

Wenn eine Sprache als Einheit konstruiert wird und sie in globalisierte Zusammenhänge eingebunden ist, bleibt es nicht aus, dass sie den Mechanismen eines weltweiten Marktverhaltens unterworfen wird. Aus einer abstrakten, kollektivkognitiven Konstruktion wird so ein sehr konkretes Produkt, das entsprechend der globalen ökonomischen Logik behandelt oder sogar gehandelt wird. Es erhält Wertzuschreibungen - materielle wie immaterielle - und es wird in einen Wettbewerb positioniert, nicht zuletzt mit anderen Sprachen. Als konkurrierendes ,Produkt' des Spanischen am weltweiten Sprachenmarkt ist dabei nicht unbedingt an vorderster Stelle das Englische zu sehen, weil es vielerorts als primäre Fremdsprache und globale Lingua Franca längst einen eigenen Platz und eine klar umrissene Rolle erlangt hat. In vielen Gesellschaften, und sicherlich in den meisten Gegenden Deutschlands, steht das Spanische in seinen Lernmärkten eher im Wettbewerb mit dem Französischen. ${ }^{11}$ Auch diese Tatsache ist in einen globalen Zusammenhang eingebettet: Soweit man die weltweiten Sprachräume des Spanischen und des Französischen noch als abgrenzbar auffassen kann, sind sie weitgehend komplementär zueinander, vorwiegend aufgrund der kolonialen Vergangenheit. In ihren Funktionen als Fremdsprachen im Schulwesen oder in der Erwachsenenbildung scheinen Französisch und Spanisch sich aber ein Stück weit zu überschneiden, wenn es etwa um Wahlmöglichkeiten geht. Wie das Spanische in Berlin bewertet wird, im Verhältnis zu Französisch aber auch im Gesamtgefüge anderer vorhandener (Fremd-)Sprachen, soll unter anderem anhand des Rahmens von Sprachen als Ressourcen betrachtet werden.

Inwiefern eine Sprache als Ressource gesehen wird, also welcher Wert ihr zugeschrieben wird, hängt wiederum von Spracheinstellungen und -ideologien ab. Im Rahmen des ideologiebasierten Language Making erlangt eine Sprache also in den Augen der Sprachgemeinschaft selbst, aber auch außerhalb, einen gewissen Wert. Dieser hängt von unterschiedlichen Faktoren ab, beispielsweise der kommunikativen Reichweite, häufig geknüpft an die Größe oder Verbreitung der Sprachgemeinschaft, aber auch von literarischen und anderen kulturellen Traditionen oder auch historisch-politischen Prozessen, in denen die jeweilige Sprache ein

11 Eine privilegierte Stellung kommt dem Französischen in Deutschland weiterhin in Grenzgebieten zu Frankreich, Luxemburg und Belgien zu, vgl. etwa Krämer (2018, 2019a) und die Beiträge in Lüsebrink/Polzin-Haumann/Vatter (Hg., 2017) zur Frankreichstrategie des Saarlandes. 
Einflussfaktor ist bzw. war. Die Darstellung von Sprachen als Ressourcen ist in der soziolinguistischen Literatur weithin kritisiert worden, weil sie Sprachen nicht als vielseitige Mittel von Gedankenaustausch und -festigung, kultureller Praxis, zwischenmenschlicher Verständigung und sozialer Bindung sieht, sondern sie utilitaristisch auf ihre Funktion als effektiv wirkendes Kommunikationsmittel reduziert, das insbesondere ökonomisch nutzbar gemacht werden kann (Lo Bianco 2017; de Jong et al. 2016; Ruíz 1984). Diese Kritik ist stichhaltig und überzeugend, so dass die anderen Aspekte von Sprache in den folgenden Überlegungen und in der empirischen Arbeit auch angemessen berücksichtigt werden müssen. Der Begriff Ressource ist allerdings hinreichend flexibel, um auch die nicht-ökonomischen und nicht-utilitaristischen Dimensionen von Sprache mit abzudecken. Sprache kann beispielsweise ohne Weiteres auch als Ressource mit schöpferischem Potenzial für künstlerisches Schaffen gesehen werden, oder auch als Ressource für ein verständnisgetriebenes Zusammenleben.

Dass eine ressourcenorientierte Logik für viele Lernende ausschlaggebende Argumente liefert und sie für die Formation von Spracheinstellungen äußerst wichtig ist, muss in die Betrachtungsweise von Sprachen als Ressourcen mit einer marktlogischen Perspektive in die Überlegungen mit einfließen (vgl. etwa den Ansatz der linguanomics bei Hogan-Brun 2017, zuvor auch Coulmas 1992, Grin 2003). ${ }^{12}$ Betrachtet man das Spanische tatsächlich als ein Produkt am Markt, dessen Absatz sich in der Anzahl von Lernenden niederschlägt, dann stellt sich die Frage, wer den Vertrieb dieses Produktes vorwiegend vorantreibt. Mar-Molinero (2010: 169) unterscheidet zwei zentrale Richtungen, aus denen die Verbreitung des Spanischen befördert wird: „[. . .] Spanish operates both as an 'anonymous,' top-down, public language driven by dominating institutional and government policies, and also as an 'authentic,' grassroots, bottom-up language.“ Beide Mechanismen sind global gesehen gleichzeitig vorhanden und sie wirken einander nicht entgegen, sondern sie verstärken sich. Dabei muss allerdings genauer betrachtet werden, ob einer der beiden Impulse stärker ist oder möglicherweise zuerst auftritt und den anderen nach sich zieht. Im Falle von Spanisch in Berlin oder auch in Deutschland dürfte die Bewegung von unten stärker ausgeprägt sein, wenn öffentliche und privatwirtschaftliche Akteure ihr Angebot zum Sprachenlernen an der Nachfrage ausrichten. Diese Frage wird in den folgenden Kapiteln eine Rolle spielen, wenn es um den Zeitpunkt des Sprachenlernens und den zielgerichteten Sprachlernwunsch in der Gemeinschaft der neuen Spanischsprecher^innen geht.

12 Einen ausführlichen Forschungsüberblick zu den Kontaktfeldern von Ökonomie und Linguistik bieten Vigouroux/Mufwene (2020). 
Als Ressourcen können Sprachen dann angesehen werden, wenn man in ihnen einen bestimmten Nutzen sieht. Es liegt auf der Hand, dass die Betrachtung von Sprachen als nutzbringende Ressourcen mit einer ökonomischen Perspektive das Risiko eines enorm verengten Blickes mit sich bringt: Diese Sichtweise ist stark instrumentalistisch, denn sie nimmt vor allem die Verwendbarkeit von Sprachen in den Blick. Der Nutzen einer Sprache kann materiell sein, etwa in Form von verbesserten Chancen am Arbeitsmarkt, höheren Löhnen durch Zusatzqualifikation oder größere Vermarktungschancen für ein Produkt durch den Einsatz zielgruppengerechter Sprachen. Daneben kann aber auch ein immaterieller Nutzen in Sprachen erkannt werden, beispielsweise erweiterte Möglichkeiten zur Kommunikation mit anderen Menschen, leichteren Zugang zu Informationen oder eine Ausweitung von kulturellen Erlebnissen. Die Größe der Sprachgemeinschaft kann dabei ein wichtiger Faktor sein: „A large speech community offers a wide spectrum of communicative opportunities and allows its members to realize these opportunities at many different places, entailing not only differentiation but also mobility“ (vgl. Coulmas 1992: 62). Der Übergang zu einer eher hedonistischen, also quasizweckfreien Sichtweise auf Sprachkenntnisse zum reinen Vergnügen an der Sprache selbst sind hierbei fließend. Im letzteren Fall bedient die Sprache emotive oder ästhetische Bedürfnisse, sie hat dann also vor allem einen affektiven Wert. In den weiter unten folgenden Befragungen und Datenanalysen werden die drei hier beschriebenen Dimensionen als Komponenten von Spracheinstellungen leitend und gliedernd sein: Die materielle, die instrumentelle und die affektive Ebene der Einschätzung des Spanischen ergibt, mit all den Überschneidungen zwischen den drei Ebenen, ein breites Bild der Bewertung der Sprache als Ressource. Hier zeigt sich, dass ein absolutes „detachment of language-as-skill from language-as-identity“, wie es Heller (2010: 110) in der linguistischen Anthropologie diagnostiziert, letztendlich keinen Bestand haben kann.

Die einseitig materialistisch-ökonomistische Betrachtung von Sprachen kann durch instrumentalistische und affektive Annäherungen ausgeglichen werden. Zugleich lässt sich selbstverständlich auch mit Sprachen mit einer kleineren Anzahl von Sprecher`innen die Bandbreite von „communicative opportunities“ erweitern. Für einzelne Lernende kann daher die Wahl einer ,kleineren' Sprache für bestimmte kommunikative Ziele einen weitaus höheren Wert besitzen, als das Erlernen einer ,großen' Sprache. Heller (2010: 102) weist darauf hin, dass auch in diesem Fall letztendlich die Sprache von einer kommodifizierenden Sichtweise erfasst werden kann, denn sie wird unter Umständen gesehen als „useful as added value for niche markets and for distinguishing among standardized products that have saturated markets.“ Genau deshalb muss die Einstellungsforschung zum Fremdsprachenlernen jeweils individuelle Motivationen und subjektive Einschätzungen erfassen, denn die vermeintlich objektive Bestimmung des ,Wertes“ einer 
Einzelsprache als Ressource führt notwendigerweise zu enormen Vereinfachungen. Der Gewichtung der drei genannten Ebenen, ihrem Beitrag zum Gesamtbild des Spanischen, gilt daher bei den folgenden Untersuchungen ein Hauptinteresse.

Es darf dabei nicht in den Hintergrund geraten, dass alle drei Ebenen und damit auch die affektive - eng verknüpft sind mit der Kommodifizierung einer Sprache wie etwa des Spanischen. Wird eine Sprache mit hohem affektivem Wert belegt und damit beispielsweise als Mittel der Identitätsbildung eingesetzt, so kann sie damit wiederum als wertschaffendes Gut eingesetzt werden (Heller 2010: 103). Als Teil eines nach innen wie außen wirkenden Selbstbildes kann die Bindung an eine Sprache wirksam werden, wenn eine Person darüber eine bestimmte Position im sozialen Gefüge erlangt, beispielsweise indem sie Zugehörigkeit geltend machen kann. Eine hohe affektive Bindung vieler Menschen an eine Sprache wiederum kann bei der materiellen Vermarktung einer Sprache als Lernprodukt durch gezieltes Ansprechen der emotiven und ästhetischen Assoziationen gewinnbringend eingesetzt werden.

Der Nutzen einer Sprache als Ressource kann den Sprechenden selbst zugutekommen, indem sie die materiellen oder immateriellen Vorteile der Sprachkenntnisse vorwiegend bei ihnen selbst liegen. Man kann diese Sprachen dann als interne Ressourcen auffassen, die bei den Sprecher*innen selbst liegen. Der Gegenpol dazu sind Sprachen als externe Ressourcen, wenn davon Menschen oder Instanzen profitieren, die selbst diese Sprachen nicht sprechen. Eingeschlossen sind dabei auch nicht-individuelle bzw. nicht-menschliche Akteure wie Institutionen oder Unternehmen. Beide Dimensionen schließen einander selbstverständlich nicht gegenseitig aus. Eine Ressource kann an unterschiedlichen Stellen zugleich Nutzen bringen, also in verschiedenem Grad als interne und als externe Ressource wirken. Problematisch ist dabei zudem die Tatsache, dass der Nutzen schwer messbar ist:

Savoir si ce capital humain est ou peut devenir un investissement rentable pour les entreprises d'une part ou pour l'individu d'autre part, notamment pour le locuteur d'une langue minoritaire, reste en débat et les vues ne s'accordent pas forcément. Cela est dû à la difficulté de mesurer le concept de "valeur » ou d'avantage économique (marchand et non-marchand) rattaché à la notion de capital humain. (Dubois/LeBlanc/Beaudin 2006: 17)

Je nachdem, für wie nützlich eine Sprache als Ressource gehalten wird, kommt ihr in den Augen der Sprecher`innen und auch außerhalb der Sprachgemeinschaft ein bestimmter Wert zu. Dieser Wert lässt sich nicht objektiv quantifizieren, sondern höchstens relational im Vergleich zu anderen Sprachen einschätzen. Im übertragenen Sinne treten Sprachen dadurch in einen Wettbewerb auf einem Markt. Mit Bourdieu gesprochen: „[I]1 y a d'abord la valeur distinctive qui résulte de la 
mise en relation que les locuteurs opèrent, consciemment ou inconsciemment, entre le produit linguistique offert par un locuteur socialement caractérisé et les produits simultanément proposés dans une espace social déterminé“ (Bourdieu 2001 [1982] : 61). Zu den locuteurs als sehr allgemein gefasste Gruppe gehören selbstverständlich auch die Lernenden und Lehrenden.

Welche Sprachen welche Wertzuschreibung erhalten, welche Einstellungen und Assoziationen an sie geknüpft werden, welche bevorzugt und welche vernachlässigt werden, all das entscheidet sich in einem ständigen Prozess des Aushandelns - sowohl in der konkreten Gesprächssituation in mehrsprachigen Zusammenhängen, wenn aus dem sprachlichen Repertoire eine Wahl für die angestrebte Kommunikation getroffen werden muss, als auch im abstrakten Zusammenhang wenn der Status von Sprachen in Gesellschaften, Ländern, Unternehmen oder sozialen Gruppen bestimmt wird.

Neben dem Markt im übertragenen Sinne sind beim Fremdsprachenlernen auch tatsächliche Marktkräfte, materielle Werte und ökonomische Mechanismen im Spiel. Wenn Sprachkurse kommerziell angeboten werden, Preise für sie festgelegt werden und ein Zusammenspiel aus Angebot und Nachfrage nach unterschiedlichen Fremdsprachenangeboten entsteht, dann wird das Sprachenlernen und -lehren wenig anders behandelt als die Inanspruchnahme anderer Dienstleistungen (Becker 2018: 188-192, Schnitzer 2012: 161). Konsequenterweise werden Sprache auch als Produkte beworben, um möglichst viele Lernende zu gewinnen. Dies tun nicht nur die Bildungsanbieter selbst, sondern auch Staaten, Regionen oder Vereinigungen, die beispielsweise auswärtige Kultur- und Sprachpolitik betreiben. Interessanterweise wird als Vermarktungsargument häufig wiederum der ökonomische Wert der Sprache angeführt. Valle/Villa (2007: 99) paraphrasieren die Argumentation: „[E]l español es una lengua útil y rentable y su conocimiento puede constituir un valioso recurso económico para quien la posea.“ Ein Produkt auf dem Markt wird auf diese Weise mit seinem eigenen Marktwert beworben. Ob das Argument von den Lernenden aufgegriffen wird, können die Resultate der Befragungen in den späteren Kapiteln zeigen.

Trotz aller Parallelen zu ,gewöhnlichen' Handelsprodukten fügen sich Sprachen nicht ohne Weiteres in die Logik von Markt und Wettbewerb ein. Grin (2006: 84) diagnostiziert insbesondere mit Blick auf sprachpolitische Maßnahmen, die aus einer rein ökonomisch getriebenen Logik durchgeführt werden: „[E]very form of market failure occurs when it comes to the provision of linguistic diversity." Zwei zentrale Widersprüche werden sichtbar, wenn man marktwirtschaftliche Kategorien auf Sprachenlernen und Mehrsprachigkeit anwenden möchte.

Der erste Widerspruch liegt in der Sichtweise des Wettbewerbs zwischen Sprachen. Dies liegt nicht nur an der naheliegenden Tatsache, dass Sprachen selbst keine Akteure sind und daher nicht in einen Wettbewerb treten können. 
Auch Produkte anderer Märkte sind schließlich selbst keine Wettbewerber, so dass hinter der Darstellung von Wettbewerben zwischen Produkten in erster Linie ein metaphorischer Bezug zum Wettbewerb zwischen den Anbietern steht. Vielmehr entsteht der Wettbewerb zwischen Sprachen dadurch, dass Lernende sich für den Einsatz bestimmter Mittel zugunsten bestimmter Sprachen entscheiden müssen und dieselben Mittel logischerweise nicht mehr für andere Sprachen einsetzen können (Grin 2010: 72). Die eingesetzten Mittel können materiell sein (etwa Kosten für die Kursteilnahme, Lernmittel, Anreise) oder immateriell (Zeit, physische oder psychische Anstrengung und Erschöpfung). Gerhards (2010: 19) spricht hier ebenfalls in einer stark von Marktlogik geprägten Terminologie - von „trade offs“

Geht man davon aus, dass die Zeit, die man zum Lernen einer bestimmten Sprache benötigt, nicht für andere Lernaktivitäten zur Verfügung steht, dann ist der Nutzen des Erlernens einer Sprache ins Verhältnis zu setzen zu dem Nutzen, den man hat, wenn man dieselbe Zeit für eine andere Aktivität aufbringt. Wir gehen der Einfachheit halber davon aus, dass die Entscheidung, was man lernt, nur zwischen verschiedenen Sprachen erfolgen kann.

(Gerhards 2010: 19-20)

Erneut tritt hier die utilitaristische Dimension zutage, nämlich der Nutzen des Sprachenlernens gegenüber anderen Aktivitäten - oder, als übertragbarer Fall, der Nutzen des Lernens einer Sprache A anstelle einer Sprache B. Im wirtschaftswissenschaftlichen Sinne kann man darin die Übertragung des Konzepts der Opportunitätskosten sehen, also die entstehenden Nachteile, die sich bei der Auswahl einer bestimmten Option durch den Verzicht auf die Vorteile einer Alternative ergeben. Im Sinne von Coulmas (1992: 77) bedeutet das, allgemeiner ausgedrückt, dass „[. . . ] the value of a language is determined in relation to that of others. “ Ein solcher Wert entsteht beispielsweise, wenn anstelle einer Sprache eine andere genutzt werden kann, die mindestens einer beteiligten Person die Kommunikation erheblich erleichtert und die dadurch ggf. auch die Erlangung weiterer materieller Vorteile ermöglicht: „Die Verfügbarkeit von Sprache, das Agieren-Können in der Muttersprache, senkt, rein materiell gesehen, die Kosten einer wirtschaftlichen Tätigkeit [. . .]“ (Schnitzer 2012: 161). Coulmas versteht value eher im engeren, materiell-ökonomischen Sinne, man kann die Aussage aber ohne Weiteres ausdehnen auf den gesamten Nutzen einer Sprache als kommunikative Ressource. Der Begriff value ist dann eher als Bewertung beispielsweise durch die Lernenden oder Sprecherinnen zu sehen denn als materiellen Wert.

Besonders evident ist die relative Bewertung von Sprachen untereinander im Bereich schulischer Fremdsprachen, für die in klassischen Lehrmodellen nur eine bestimmte Anzahl von Wochenstunden zur Verfügung steht. Die finanzielle Dimension steht dagegen vor allem bei kostenpflichtigen Lernangeboten etwa von Sprachschulen im Mittelpunkt. Dasselbe gilt bei der Frage, wie viel öffentliches Geld für 
die Förderung von Lernangeboten bestimmter Sprachen aufgewandt werden soll (Coulmas 1992: 101-104).

Trotz alledem ist die Konkurrenz zwischen den Sprachen in diesem Sinne nicht so klar geordnet, wie es auf den ersten Blick erscheint. Der Einsatz von Mitteln für Sprache A bedeutet nicht automatisch, dass derselbe Umfang von Mitteln nicht für Sprache B zur Verfügung steht, auch wenn dies in öffentlichen Debatten um den Umfang von Schulfächern oder nach der Förderung bestimmter Sprachen oft so dargestellt wird. Anders ausgedrückt: Die Opportunitätskosten beim Erlenen von Sprache A entsprechen nicht der vollen Höhe des Nicht-Erlernens von Sprache B. Der „trade off“ im Sinne Gerhards' ist nicht vollkommen symmetrisch. Sprachen schließen einander nämlich nicht komplett gegenseitig aus, wie sich besonders deutlich anhand additiver Mehrsprachigkeit sehen lässt. Geht man von Deutschen als Erstsprache aus, kann der Aufwand für das Erlernen des Niederländischen als nah verwandte Sprache A nicht ohne Weiteres gleichgesetzt werden mit dem Verzicht auf das Erlenen des Spanischen als weniger nah verwandte Sprache B. Dasselbe gilt auch bei einer Abfolge von Sprachen, wenn etwa zunächst Sprache A statt B gelernt wird, B aber später nachgeholt wird. Das Lernen einer Sprache B nach Sprache A kann weniger Zeit und damit unter Umständen auch weniger Geld kosten, beispielsweise wenn sie mit Sprache A nah verwandt ist, also leichter und schneller zu erwerben ist. Gerade die häufig konstruierte Konkurrenz zwischen Spanisch und Französisch ist daher keine absolute (vgl. hierzu auch Bär 2017: 87). Der Gegensatz ist ein Konstrukt, nicht zuletzt weil sich der Erwerb der beiden eng verwandten romanischen Sprachen sehr gut gegenseitig unterstützt. Selbst wenn keine nahe Verwandtschaft vorliegt, kann bereits die Tatsache, dass jemand Erfahrung im Sprachenlernen hat und die eigenen Lernstrategien gut einschätzen kann, einen Vorteil beim Erlernen weiterer Sprachen bringen und somit den Ressourceneinsatz verringern. Wenn der Aufwand von Zeit und Geld für Sprache A also den Aufwand für Sprache B erheblich verringert, kann zumindest von einem absoluten Wettbewerb zwischen zwei Sprachen keine Rede sein.

Der zweite Widerspruch zwischen Marktlogik und Sprachenlernen liegt einem Grundmechanismus des Language Making, nämlich der Schaffung von abgrenzbaren Konzepten. Wenn Sprachen als Produkte auf einem Markt gesehen werden, dann werden sie als Einheiten angeboten. Sprachen erscheinen zum Beispiel in Kurskatalogen, aufgeführt unter ihren einzelnen Bezeichnungen, zudem gestückelt in Unterrichtseinheiten mit einer bestimmten Anzahl Stunden und einzelne Kurse, die meistens in Sprachniveaus nach dem europäischen Referenzrahmen zerlegt werden. In vielerlei Hinsicht erscheinen Sprachen so einteilbar, portionierbar.

All diese Mechanismen widersprechen der Tatsache, dass eine Sprache als variable Praxis keine abgeschlossene Einheit bildet und deshalb im Prinzip gar nicht als Produkt ,aus einem Guss‘ angeboten werden kann. Eine Sprache als 
Produkt lässt sich also nur dann stückweise verkaufen, wenn sie als einheitlich konstruiert wird. Hier spielt erneut die Konzeptionalisierung von Sprache als normengebundenes Objekt eine Rolle, das durch Language Making repräsentativ fassbar gemacht wird. Insbesondere der Standardsprache als vermeintlich klar abgegrenztes Set von Sprachformen kommt eine herausragende Stellung beim Sprachenlernen zu, denn auf sie richtet sich häufig die Lernabsicht und die Vermittlungsbemühung auch der Lehrenden. ${ }^{13}$ Großen Einfluss können bei der Vermittlung des Konzepts normierte Einzelsprache die kodifizierenden Referenzquellen wie Grammatiken, Wörterbücher und Lehrwerke spielen. ${ }^{14}$

Als einer der größten und weltweit tätigen Anbieter von Spanischunterricht stellte beispielsweise das Instituto Cervantes noch vor wenigen Jahren auf der Internetseite seiner Aula Virtual de Español ganz klar heraus, dass in seinem Angebot für Online-Kurse die Vermittlung des europäisch-kastilischen Standards des Spanischen im Mittelpunkt des Unterrichts stehe, weil dieser als ,neutral' anzusehen sei (Mar-Molinero 2010: 171, vgl. auch Mar-Molinero 2006: 85-86). Das Institut schloss damit an die lange Tradition Spaniens an, das Kastilische zum Kern einer sprachlich homogenen Nation auszubauen - ein Kern, der in die nicht-kastilischsprachigen Regionen und auch in die Kolonien hineinwirken sollte (García 2011: 670). Inzwischen ist der Hinweis auf den Webseiten des Instituto Cervantes nicht mehr zu finden. Stattdessen wird nur noch auf eine weitaus größere Bandbreite von Diversität hingewiesen, die sich über mehr als nur sprachliche Variation erstreckt. So heißt es auf der Online-Lernplattform des Instituto Cervantes unter der Kategorie fundamentos didácticos:

En los materiales de los cursos en línea del Instituto Cervantes se pretende dar una imagen realista de la sociedad contemporánea, en la que se representan las diferencias de forma positiva y se fomenta el conocimiento de las minorías. Esta visión actualizada se plasma en la concepción general de los contenidos culturales de los temas y cursos y, más concretamente, en la creación, selección y revisión de los vídeos, las locuciones, los textos, las ilustraciones y las fotografias. ${ }^{15}$

(Hervorh. im Original)

Lediglich hinter der Auswahl von Ausdrücken und Redensarten sowie von Texten verbirgt sich noch ein Hinweis auf möglicherweise unterschiedliche Erscheinungsformen des Spanischen und eine Form von Variation. Es zeigt sich ein nach außen

13 Vgl. etwa De Wilde (2019) zu den standardsprachideologischen Hintergründen in der universitären Fremdsprachenlehre am Beispiel des Niederländischen.

14 Vgl. Greußlich (2015) zum Umgang mit dem plurizentrischen Charakter des Spanischen in der Nueva gramática de la lengua española; eine kritische Würdigung des Konzepts der Plurizentrik für die Lehre des Spanischen als Fremdsprache liefert auch Corti (2019: 102-114).

15 https://ave.cervantes.es/informacion_general/fundamentos_didacticos.html (Letzter Aufruf: 10. August 2020). 
sichtbarer Schritt dahingehend, die Sprache nicht mehr unmittelbar als landesgebunden darzustellen, sondern als global attraktive Praxis (García 2011: 678). In den Lehrmaterialien selbst wird Variation und der plurizentrische Charakter des Spanischen zumindest gelegentlich dargestellt, wenn auch der Schwerpunkt weiterhin deutlich auf der europäischen Norm liegt:

Dado que el CVC [Centro Virtual Cervantes, PK] es una institución dirigida y diseñada en España, los diversos fenómenos diatópicos siempre se refieren a sus correspondientes del español peninsular. [. . .] Sin embargo, se puede afirmar que los materiales analizados se orientan hacia un punto de vista de apertura panhispánica.

(K. Leonhardt 2012: 325)

Welche Eigenschaften das angebotene Produkt hat, d.h. welches Spanisch genau gelehrt wird, ist ansonsten für die Kundschaft praktisch nicht mehr im Voraus nachvollziehbar, ohne die Kursinhalte oder Lernmaterialien zu kennen und sie anschließend anhand variationslinguistischer Kenntnis einordnen zu können. Die Qualitäten des Produkts ,Spanisch` bleiben damit gänzlich einem unausgesprochenen und nicht näher beschriebenen Konsens zwischen Lehrenden und Lernenden überlassen: Man ist sich ohne weiteren Abgleich auf einer sehr abstrakten Ebene einig, was mit ,Spanisch' gemeint ist bzw. man geht vielmehr davon aus, dass die Vorstellungen von der Sprache einigermaßen deckungsgleich sind. Corti (2019: 102) weist hier auf eine zentrale Frage für die Fremdsprachenlehre des Spanischen hin, die zugleich auch in der Forschung berücksichtigt werden muss: „En el caso de ELE, sería necesario atender al posicionamiento de aprendientes y enseñantes respecto a la normatividad.“ Das Bild des ,Spanischen“ kann dabei durchaus individuell sehr unterschiedliche Ausprägungen annehmen, von einer fortbestehenden Anerkennung der Normkraft des peninsularen Spanischen über eine breit variierende Plurizentrik bis hin zu einer Art ,panhispanischem' Kern der Sprache (Oesterreicher 2000, Lebsanft 2004, Lebsanft/Mihatsch/Polzin-Haumann 2012, Paffey 2012: 150-157). Beruht der vermeintliche Konsens auf unterschiedlichen Annahmen, entspricht also das gelehrte Spanische nicht den Erwartungen der Lernenden, weil diese beispielsweise mehr lateinamerikanische Strukturen erwarten, dann kann dieser Unterschied in den Vorstellungen des Produkts erst nachträglich offengelegt werden. ${ }^{16}$ Die verschränkte Vermittlung von Sprachformen und kulturellen Werten, Assoziationen oder Weltanschauungen, die gerade in der auswärtigen Sprachund Kulturpolitik (para)staatlicher Akteure präsent ist, bleibt dabei unausgesprochen und tritt in den Hintergrund. Dabei gilt ganz deutlich: „A language is not a

16 Im Unterschied dazu werden beispielsweise gelegentlich explizit Kurse für europäisches oder brasilianisches Portugiesisch angeboten. 
widget that can be manufactured, bought or sold with no other values attached to it“" (Hogan-Brun 2017: 34).

Mar-Molinero und Paffey (2011) werfen dabei die Frage auf, inwiefern die zunehmende Verbreitung des Spanischen mit Begriffen wie „linguistic imperialism“ zu erfassen ist, wenn damit zugleich Spracheinstellungen und ein weiter gefasstes Kultur- und Wertebild vermittelt wird. Auch hier ergeben sich Parallelen zu einer globalen Marktlogik, etwa bei der weltweiten Verbreitung großer Marken und bekannter Produkte, die ebenfalls mehr oder weniger offen mit einem bestimmten Wertegerüst weitergetragen werden.

Inwiefern Lernende überhaupt das Bedürfnis haben, eine bestimmte Form des Spanischen zu erwerben, wird in den Untersuchungen in den Kapiteln 7 und 8 angesprochen. Ist dies nicht der Fall, wäre es wahrscheinlich angebrachter, von einem Produkttyp zu sprechen, der von Anbietern wie Kundschaft unter dem Sammelbegriff Spanisch gehandelt wird, ohne dass die konkrete Erscheinungsform notwendigerweise exakt identisch sein muss oder die Kundschaft eine ganz bestimmte Erscheinungsform alternativlos erwartet. Anders ausgedrückt: Es ist unklar, ob exakt dasselbe Produkt von verschiedenen Anbietern auf den Markt gebracht wird, oder ob vielmehr unterschiedliche Ausprägungen desselben Produkttyps nachgefragt werden. Dies hat auch Auswirkungen auf die Mechanismen des Wettbewerbs, nämlich auf die Frage, ob die Angebote tatsächlich als austauschbar angesehen werden. Ist beispielsweise das Spanische in einem Volkshochschulkurs dasselbe Produkt wie im Kurs einer privaten Sprachschule, das lediglich in einer anderen ,Verpackung“ dargeboten wird?

Nicht zuletzt muss bei alldem auch beachtet werden, dass die Lernmärkte für Sprachen ohnehin keine perfekten Märkte sind, wie man sie bei anderen Produkten eher vermutet (Coulmas 1992: 79-85). Dies wird bereits dadurch deutlich, dass ein großer Teil der Sprachvermittlung durch das öffentliche Schulwesen geleistet wird und auch weitere staatlich finanzierte oder geförderte Akteure wie Hochschulen, Volkshochschulen, staatliche Einrichtungen wie das Instituto Cervantes und viele mehr auf dem Markt aktiv sind. Sie federn das ökonomische Spiel von Angebot und Nachfrage zu einem bedeutenden Teil ab und sorgen dafür, dass der Erwerb von zentralen Gütern, wie es Sprachkenntnisse sind, nicht ausschließlich von den materiellen Möglichkeiten der einzelnen Person abhängig ist. Die bestehende sozioökonomische Ungleichheit auch im öffentlichen Bildungswesen bleibt dabei selbstverständlich dennoch weiterhin äußerst wirksam.

Die Bewertung des Nutzens einer Sprache als Ressource hängt häufig davon ab, wie groß die Anzahl der Muttersprachler^innen ist, welches Bild von ihnen gepflegt wird oder wie präsent diese in der Wahrnehmung sind. Das folgende Kapitel widmet sich deshalb einem kurzen Überblick zum Spanischen als Muttersprache in Berlin, bevor das Spanisch als Fremdsprache näher beleuchtet wird. 